\title{
Predicting Relationship Satisfaction from Dispositional Optimism Amongst young adults in Mumbai city
}

\author{
Anuja Deshpande ${ }^{1}$ \\ Gauri Sarda ${ }^{2}$ \\ ${ }^{1}$ Assistant Professor, Department of Psychology, Smt. Maniben Nanavati Women's College, \\ Mumbai. \\ ${ }^{2}$ Research Scholar, Savitribai Phule Pune University. \\ E-mail - anuja185@gmail.com
}

\section{ABSTRACT}

This study was conducted to see whether it was possible to use dispositional optimism to predict satisfaction with romantic relationships in young adults in Mumbai city. Data was collected from 92 participants using purposive and snowball sampling methods. All participants of both genders who were aged between 20 - 30 years and were in a relationship for at least one year before data collection. Statistical analysis suggested that dispositional optimism was a highly significant predictor of relationship satisfaction among the research participants. The reasons and implications of these findings are discussed.

Keywords: Optimism, Relationship Satisfaction, Young Adults.

\section{INTRODUCTION}

Development theory posits that developing and maintaining healthy romantic relationships is one of the essential tasks for young adults [1]. Romantic relationships and the factors that influence the health of these relationships are among the most discussed topics in popular psychology, as well as in advice forums for young adults. It thus stands to reason that serious research into the factors that impact the quality of romantic relationships needs to be considered as a subject for scientific research as well. Optimism has long been considered a valuable trait across human activity. It has found mention in philosophical writings as well as in modern scientific literature [2]. Optimism has been considered the elixir that wards of the ill effects of stress and strife in a number of stories. This belief in the importance of optimism seems to be substantiated by scientific research as well. A number of researchers have also found evidence that suggests that optimism helps insulate relationships from the impact of stressors [2]. Authors [3] reviewed the factors that helped first-time parents cope with the stress associated with having a child. They found that optimism about the partner and optimism about the process of adjustment to their new roles helped new parents relate better to each other and to the novel experiences. Optimism, with a number of other factors also helped reduce the chances of separations 
and divorce in the immediate period after child-birth. Optimistic partners thus seemed to be able to cope with difficult events and changes in the relationship more successfully.

Researchers [4] found that when an individual idealised their partner and experienced optimism about the partner and the relationship, they were more satisfied, perceived their partner as showing greater love and trusted their partner more. They also experienced less conflict in their relationships and were more positive about the development of the relationship. This effect was found for both married and dating couples with varying relationship lengths. Over time, the couples who shared optimistic outlooks (among other relationship illusions) were more likely to have successful relationships [4]. The researchers also found that this effect was correlated to the strength of the illusions initially reported. In another study, it was found that when people were optimistic, they were more likely to use cooperative problem solving techniques to resolve issues in romantic relationships. They also found that optimistic people reported increases in their satisfaction with their relationship over a two year period. Thus, optimistic persons were less likely to emphasise conflict, and were also less likely to make their partners feel defensive when responding to a conflict [5]. It is likely that the cooperative process of resolving conflicts also led to a more involvement in the relationship and in the partner - thus leading to a more optimistic and positive outcome in the long run [2].

Optimism need not be considered as a general state, and can be specific to relationships. This can be affected by past experiences as well as the experiences of others romantic encounters. It has been found that people tend to exhibit two kinds of optimism about romantic relationships. These include optimism about the future of the relationship and optimism about the likelihood and success of marriage in the relationship. They found that the participants' own experiences in dating and romantic relationships were strong predictors for the first kind of optimism, but not the latter. Success of the parents' marriage and the extent to which their parents had happy marriages was found to be able to predict optimism about the likelihood and success of marriage. Thus, optimism about a particular relationship can be affected by a complex inter-play of factors from the person's background [6]. It has also been found that parental divorce makes participants less optimistic about their own relationships and future marriages. Participants in this study - whether with divorced parents and from intact homes - did not differ (or were matched) in dispositional optimism; underlining the role of parental divorce in the child's ability to form trusting relationships. The researcher also found that this effect was mediated by people's assumptions about how benevolent people are in general. Thus, the more the participants with divorced parents believed in other people's benevolence, the more likely they were to be optimistic [7].

In spite of their particular relationship backgrounds, some people just tend to be more optimistic about all aspects of their lives as compared to others. Dispositional optimism itself is associated with a number of personal and developmental experiences. In turn, it impacts a number of experiences and outcomes for the individual. It was found that optimistic persons were more likely to experience satisfaction with their lives across different areas. On the other hand, they also found that maladjusted persons were less likely to experience optimism; although there seemed to be no discernable relationship between life satisfaction and maladjustment. Well-adjusted individuals, on the other hand, did seem to be more optimistic overall [8]. Thus, it seems that well-adjusted individuals have a disposition conducive to 
experiencing optimism; and this optimism in turn leads them to feel satisfied with life. It was also found that an optimistic outlook enabled people to find strength in the most trying of events. They studied women with breast cancer, and found that patients who were more optimistic felt they had more social support and thus experienced more psychosexual well-being. These women were more likely to feel that they were feminine, attractive, and sexually desirable in spite of their illness and were also less likely to experience distress about the illness immediately after treatment as well as over the months of follow-up [9]. These results suggest that dispositional optimism is a valuable trait, regardless of the life experience.

This is true of romantic relationships as well as difficult experiences. Optimistic persons are not only more satisfied with their relationships if they perceived that they received social support; but also deal with conflict in a constructive manner. They also reported better conflict resolution as compared to pessimists. People who were optimistic in the beginning of their relationships had better and more successful relationships over time and also contributed more to other aspects of the relationship. Thus, optimistic people not only dealt with conflicts better, but also laid the ground for a more stable and healthy relationship [10]. Thus, it seems that optimistic persons take steps and make sacrifices in a relationship in order to enhance the quality of the relationship, or because they believe that their partner would benefit from the action. On the other hand, pessimistic individuals are more likely to make sacrifices because they want to avoid a negative experience like and argument. Researchers conducted a series of studies to explore the contribution of motives to the quality of the person's relationship. They found that when a person made a sacrifice for their partner due to approach motives (wanting to help the partner or add to the relationship) they experienced enhanced personal wellbeing and better quality relationships. On the other hand, when people made a sacrifice through an avoidance motive (wanting to avoid a negative event) they experienced decreased wellbeing and relationship quality [11]. The researchers also found that an individual's perception of whether the partner was using an approach or an avoidance motive (when making sacrifices) was significantly associated with enduring quality of the relationship.

It seems that it is helpful to view ones partner through rose-tinted glasses. Research has found that in the most successful and happy relationships, people liked their partner more than the partner liked themselves [12]. These participants viewed their partner as more positive on a number of attributes as compared to the partner's self - evaluation on the same attributes. Such individuals' assessment of their partners matched their notions of the ideal partner as well as their own ideal self-image. This was found to be true of both dating couples and married couples, suggesting that the bias towards a partner was valuable for happy relationships regardless of how long the couple had been together. In a doctoral dissertation, Dicke [13] found that when both partners in a romantic relationship were optimistic about their future, the relationship felt more satisfying to both partners. Optimist - optimist couples also used conflict resolution strategies that added to the strength of the relationship - such as integrating and obliging conflict resolution strategies. Optimistic participants also predicted that they would sustain their relationship longer as compared to pessimistic participants. When only one partner in a relationship was an optimist, the relationship was judged as less satisfying by the couple (by averaging the responses of both partners) as compared to the ratings given by the optimist - optimist couples; while the least satisfying relationships were reported by couples where both partners were 
pessimistic. Couples where both partners were pessimistic were most likely to use dominating tactics to conflict resolution. Dicke also found that all individuals - regardless of whether they themselves were optimists or pessimists - preferred a partner who was an optimist.

This literature suggests that optimism and relationship satisfaction are related to each other. Most of this research was conducted in western countries the researchers wished to see if the same relationships among these variables held true in metropolitan India. . Given the heavy cultural bend towards not admitting to dissatisfaction in marital relationships; the researchers chose to study only non-married participants; or participants who were not considering marriage immediately. The proposed hypothesis was that it was possible to predict relationship satisfaction from dispositional optimism among young adults in Mumbai.

\section{METHODOLOGY}

This study was carried out in the metropolitan city of Mumbai and young adults were deemed the target population. Thus, the research information was collected from a sample of this predetermined population - i.e. - young adults in the city of Mumbai. Care was taken to include participants who were not married, or about to be married. All the information including demographic information and responses to the used instruments was collected from the respondents at one time. All participants were asked to respond to the instruments keeping in mind their present romantic relationship.

Participants were initially provided with some information about the study, and informed consent was taken before data collection. All scales used were self administered and easy to understand. Nevertheless, participants were assured of help in resolving their doubts as well as complete anonymity and confidentiality. To this end, names and addresses were not collected in the demographic profile.

The sample $(n=92)$ consisted of 39 males and 53 females from Mumbai. The participants were included on the basis that they were in steady relationship for more than one year at the time of data collection. The age group of the sample ranged from 20 - 30 years and the mean age obtained was 23.72 (SD = 2.034). Purposive sampling was initially used as the participants were sought from the college going students who were dating. Further data was collected using the Snowball sampling technique in order to get other suitable respondents from amongst initial participants' acquaintances.

Two instruments have been used to collect the data in the research:

1. The Relationship Assessment Scale was developed by Susan Hendricks [14] as a tool to measure relationship quality. The scale is highly reliable and valid; and has been shown to be highly correlated with longer measures of relationship quality. The Relationship Assessment Scale has been shown to be both a reliable and a valid measure of relationship satisfaction by multiple researchers [14-16]. The scale is a seven-question Likert-scale measure that deals with a single factor or global construct - i.e. - relationship satisfaction. Each item had five possible responses, though each item had slightly different response options. The items also showed good inter-item correlation, the mean inter-item correlation for the original sample being 0.49 [14]. The test-retest reliability was found to be 0.85 . The Relationship Assessment Scale was also tested for validity using several 
different tests. The loadings were in the moderate range from 0.49 to 0.79 , suggesting the measure is reasonably unidimensional.

2. The Life Orientation Test - Revised (LOT-R) [17] is a 10-item scale, with four filler items and six scale items. LOT-R Total scores are calculated by summing the three positively worded and three negatively worded items (these are reverse coded). Respondents are asked to indicate their level of agreement with each of the items on a 4-point scale, using the response format, "strongly agree" to "strongly disagree". This gives a possible range of 6-24, with higher scores indicating more optimism. Authors [17] report an internal reliability coefficient of 0.78 for an undergraduate sample. The corresponding internal reliability coefficient for the sample in the present study was 0.60. LOT-R Optimism (total of the three positively worded items) and LOT-R Pessimism (total of three negatively worded items) were also calculated. Internal reliability coefficients for these subscales were 0.62 (Optimism) and 0.78 (Pessimism). For this study, only the composite score was considered.

\section{RESULTS}

Ninety two participants between the ages of 20 and 30 years were included into the study, and data on their dispositional optimism and satisfaction with their present relationship were collected. Analysis shows that the mean score for optimism was 18.24 with an SD of 3.326 while the mean score on relationship satisfaction was 27.16 with an SD of 4.272 . This suggests that the participants on the whole were moderately high on optimism. The participants' relationship satisfaction was also found to be moderately high on the whole. The correlation between optimism and relationship satisfaction was found to be 0.402 , and the shared variance between the variables was $16.1 \%$. This suggests that the two variables share a moderate correlation. When optimism was used to predict relationship satisfaction, the unstandardized $B$ was 0.516 with a standard error of 0.124 ; while the standardised beta was 0.402 . The regression analysis yielded an $F$ value of 17.319 which was found to be significant at the 0.001 level $[F(1,90)=17.319 ; p<0.001]$. This suggests that dispositional optimism in young adults in Mumbai is a significant predictor of their satisfaction with romantic relationships. These results verify the hypothesis postulated and allow us to use dispositional optimism as a predictor for the extent to which an individual would be satisfied with their present romantic relationship. The details of the regression analysis have been reported in Table 1.

Table 1: Predicting Relationship status from Optimism

\begin{tabular}{cccccc}
\hline & Sum of Squares & df & Mean Square & $F$ & Sig. \\
\hline Regression & 267.984 & 1 & 267.984 & 17.319 & 0.001 \\
\hline Residual & 1392.570 & 90 & 15.473 & & \\
\hline Total & 1660.554 & 91 & & & \\
\hline
\end{tabular}

Dependent Variable: Relationship Satisfaction; Predictors: Optimism

\section{DISCUSSION}

It can be seen from the results that optimism does indeed predict satisfaction with romantic relationships in young people in Mumbai. The correlation value 
of 0.402 between relationship satisfaction and optimism indicated that there is a $16.1 \%$ overlap in the variance shared by these variables. This suggests that optimism seems to be a reasonable predictor of the extent to which the participants were satisfied with their relationships; and thus verifies the stated hypothesis. Thus, optimism seems to be strongly associated with relationship satisfaction in young adults and college students. These findings can also be understood in context Dicke's [13] analysis that suggested that optimists were more certain that they would have successful relationships as compared to pessimists. When both partners in a relationship are optimists, both are likely to feel satisfied with their relationship and thus, to believe that they will have a successful relationship. Conversely, it also seems intuitive enough that when an individual feels satisfied with the quality of the relationship, they are more likely to believe that the relationship would be successful and that they would continue to experience positive events as the relationship progresses.

The present results establish that optimistic persons tend to be more satisfied with their romantic relationships across cultural and age demarcations. There seem to be a number of factors that can explain the pervasive nature of this phenomenon. As suggested by authors [5], people with an optimistic disposition tend to use more constructive means when they need to resolve conflicts. Also, they are more likely to make sacrifices to enhance the wellbeing of the partner or the relationship than to avoid a negative event [11]. Thus, an optimist would be motivated to resolve the conflict thoroughly and to find a win - win solution - which in turn would lead to more satisfaction with the relationship for both the partners. They [11] also show that optimists are more satisfied with the solutions found during conflict resolution; suggesting that once a conflict is resolved, an optimist is more likely to feel satisfied with the relationship than a pessimist who is less likely to feel comfortable with solutions found. Such choices would feed back into the health of the relationship and the commitment between partners, leading to more positive experiences.

An optimist also tends to see their partner as more positive and the relationship as having more potential as compared to a pessimist [4]. This belief that their partner was loving and trustworthy would in itself enhance satisfaction with the relationship; and would also help motivate the individual to use cooperative problem solving. Such illusions would also help reduce the valence of negative events, and help them focus on positive events that feed back to further strengthen the relationship. Thus, having an optimistic disposition may be extremely valuable in the process of developing and sustaining romantic relationships. This has also been established through research which underlines the insulating value of optimism in sustaining relationships through stressful events [3]. Optimism enhances satisfaction and involvement in the relationship not only in the early stages, but throughout the life of the relationship. An optimistic individual will be able to see the best in their partner and their relationship; and thus would be more likely to predict a positive future for their relationship. Such an individual would thus experience more satisfaction with the relationship despite the odds.

The results of this study should not be used to suggest that dispositional optimism is the only predictor of how satisfied the individual is with their relationship. A large percentage of the variance in relationship satisfaction is not accounted for by optimism; suggesting that there are other mediating factors that need to be explored to understand their influence on relationship satisfaction. Further research 
in this area could try to explore the interactions among such variables that impact relationship satisfaction as well as variables that would contribute towards the eventual success of a relationship. It should also be noted that the sampling methods used here were purposive and snowball sampling; and the participants were (presumably) exclusively heterosexual. This was done in order to include only individuals who had been in a relationship for a considerable period of time and were ready to participate in the study. But this does cast some doubt about how representative these results are of the entire population. Further research in this area can use stronger data collection methods. It would also be interesting to see whether the specific nature of Indian cultural mores would affect the ability of optimism to predict relationship satisfaction among married couples, people residing in rural areas and persons with non heterosexual orientations.

\section{REFERENCES}

1. Papalia DE, Olds SW, Feldman RD. Psychosocial development in Young Adulthood. In Human Development (9th ed.) (pp. 490 - 520). New Delhi: Tata McGraw-Hill Pub. Co ; 2004.

2. L'Abate L, De Giacomo P. A developmental theory of relational competence. In Intimate Relationships and how to Improve Them: Integrating Theoretical Models with Preventative and Psychotherapeutic Applications (pp. 21 - 76). Westport, CT: Greenwood Publishing Group ; 2003.

3. Ahlborg T, Strandmark M. Factors influencing the quality of intimate relationships six months after delivery-First-time parents' own views and coping strategies. J Psychosom Obstetr Gynecol 2006;27(3):163-172.

4. Murray SL, Holmes JG. A Leap of Faith? Positive Illusions in Romantic Relationships. Personal Soc Psychol Bull 1997;23:586-604.

5. Assad KK, Donnellan MB, Conger RD. Optimism: An enduring resource for romantic relationships. J Personal Soc Psychol 2007;93(2):285-297.

6. Carnelley KB, Janoff-Bulman R. Optimism about Love Relationships: General vs Specific Lessons from One's Personal Experiences. J Soc Personal Relations 1992;9:5-20.

7. Franklin KM, Janoff-Bulman R, Roberts JE. Long-Term Impact of Parental Divorce on Optimism and Trust: Changes in General Assumptions or Narrow Beliefs? J Personal Soc Psychol 1990;59(4):743-755.

8. Khan IF, Fatima SJ. Maladjustment, Life Satisfaction and Optimism: A Correlation Study. Int J Humanities Soc Sci Invent 2013;2(5):17-26.

9. Wimberly SR, Carver CS, Antoni MH. Effects of optimism, interpersonal relationships, and distress on psychosexual well-being among women with early stage breast cancer. Psychol Health 2008;23(1):57-72.

10. Srivastava S, McGonigal KM, Richards JM, Butler EA, Gross JJ. Optimism in Close Relationships: How Seeing Things in a Positive Light Makes Them So. J Personal Soc Psychol 2006;91(1):143-153.

11. Impett EA, Gable SL, Peplau LA. Giving Up and Giving In: The Costs and Benefits of Daily Sacrifice in Intimate Relationships. J Personal Soc Psychol 2005;89(3):327-344.

12. Murray SL, Holmes JG, Griffin DW. The Benefits of Positive Illusions: Idealization and the Construction of Satisfaction in Close Relationships. J Personal Soc Psychol 1996;70(1):7998.

13. Dicke AK. Optimism and its effect on Romantic Relationships (Unpublished doctoral dissertation). Texas Tech University, Texas, USA ; 1997.

14. Hendrick SS. A generic measure of relationship satisfaction. J Marr Fam 1988;50:93-98.

15. Hendrick SS, Dicke A, Hendrick C. The relationship assessment scale. J Soc Personal Relation 1998; 15:137-142.

16. Vaughn MJ, Matyastik Baier ME. Reliability and validity of the relationship assessment scale. Am J Fam Ther 1999;27:137-174. 
17. Scheier MF, Carver CS, Bridges MW. Distinguishing optimism from neuroticism (and trait anxiety, self-mastery, and self-esteem): A re-evaluation of the Life Orientation Test. J Personal Soc Psychol 1994;67(6):1063-1078.

Acknowledgements - Nil

Conflict of Interest - Nil

Funding - Nil 